\title{
The association between dural arterio-venous fistulas (DAVFs) and meningeal-based tumors
}

\author{
M. Cunha e Sá • Cátia Gradil
}

Received: 8 November 2013 / Accepted: 8 November 2013 / Published online: 21 November 2013

(C) Springer-Verlag Wien 2013

In this article by Vellimana et al., the authors have reviewed their extensive series of patients with DAVFs, combining the experiences of two important US neurosurgical departments and looking closely at cases that concomitantly presented with a benign meningeal tumor. They have found ten such cases and describe their anatomical details, presenting symptoms and treatment strategies, and elaborating on the possible explanations for this association.

As a retrospective series, there is no matching control population, and we do not know whether in the patient population under consideration other non-tumoral pathologies have been found or in what percentage of cases; such pathologies might include, for instance, aneurysms, cavernous malformations, or AVMs, which could in theory be connected to an ongoing overpowering of the local arterial and venous circulation, internal and external carotid territories, and vertebro-basilar distribution.

One could ultimately argue that if we look into a large enough population of patients with other types of problems or tumors, we may end up finding a non-negligible number of DAVFs alongside the index problem. That does not necessarily mean that the association is anything more than spurious.

We are told that in this series combining the numbers of two experienced US neurosurgical services, no other lesions of a tumor nature were found amongst the 200 DAVFs cases reviewed. This is seemingly a difference when compared with other series looking at a lesser number of DAVFs, which have nonetheless found an association with tumors other than meningiomas. Is this a mere statistical artifact? The product of hazard? Where is the line drawn? Are DAVFs only

M. Cunha e Sá $(\bowtie) \cdot$ C. Gradil

Department of Neurosurgery, Hospital Garcia de Orta,

Almada, Portugal

e-mail: mcunhaesa@gmail.com consistently associated with meningeal-based tumors, or also with other intracranial tumor entities?

The discussion is enriched with all possibilities laying the stage for the association of DAVFs and tumors such as meningiomas, in which an intimate and often deleterious mechanical effect over the cortical veins and dural sinuses exists, thereby forcing the rerouting of blood in the vicinity of the tumor. Sinus invasion or occlusion would contribute to the venous outlet obstruction, which along with local ischemic factors might induce the opening of dormant AV dural communications, or else cause its outright occurrence.

Other relevant mechanisms may be in play, such as tumorinduced biochemical influences in thrombotic chains, as well as stimulated growth and spurting of arteries and veins, and these have been clearly pointed out by the authors.

The reported spontaneous disappearance of a DAVF after the resection of a meningioma would argue in favor of a clear causative factor between the tumor and the fistula, but is also however an insufficient argument. If we admit this association to be nonspurious, then it must rely on factors overpowering or influencing the circulation in these cases. Once the tumor is gone and the normalization of the effects on the circulation and blood volume are reestablished, the tumor(flow?)-dependent fistula would shut down on its own (a mechanism which has also been admitted as plausible for flow-related aneurysms arising in branches feeding AVMs).

Again, these arguments do not stand for all cases, given the evidence that occasionally a spontaneously disappearing AV fistula was located in an anatomic area which had nothing to do with the resected tumor nor with sinus invasion.

Also, as adequately stated in the text, this association has been found with other non-dural-based but otherwise endocrinally active tumors such as pituitary adenomas or chemodectomas.

The lack of a control population and the wavering and uncertainties in the decision-making of the patients in the 
series combine to cast further shadows on possible conclusions to be drawn from the study. Even in the presented case of the phosphaturic tumor, there was a time lag of 1 year between the initial MRI with the vascular image alongside the tentorial rim and the actual angiography highlighting the fistula. The indication to treat primarily the symptomatic lesion may be tenuous or even difficult to admit in cases where the fistula is associated with a sizeable tumor. The symptoms arising from the congestion elicited by the bulk of the tumor and its effect on the circulation locally and at a distance may mimic the symptoms one would expect to find related to the venous overpowering of the circulation caused by an AVF, or the other way around.

One of the main reasons to treat DAVFs is the presence of clear angiographic signs of congestion in the venous return channels, especially when reflux exists in the adjacent cortical veins. In cases where proximity exists between the meningioma and the DAVF, it may again be difficult to understand whether these signs of congestion are merely related to the mechanical effect of the tumor and therefore solved with its resection, or are also independently attributed to the fistula itself and therefore require a therapeutic attitude directed exclusively to the fistula.

Unless the two lesions are identified at a distance from each other, it may be difficult to clearly distinguish which are the engorged vessels commonly associated with tumors such as meningiomas, and which are outright anatomical DAVFs leading an independent existence.

Having said this, it will be similarly difficult to identify which lesion should be dealt with first. In my opinion, the right option would be to go for the symptomatic lesion first, or the one that involves potentially more danger for the patient. Of course, if both lesions are in close proximity, they should ideally be treated simultaneously.

Conflicts of interest None. 